\title{
MOLECULES IN THE MAGELLANIC CLOUDS
}

\author{
R.S. BOOTH \\ Onsala Space Observatory \\ Chalmers University of Technology \\ S-439 Onsala \\ Sweden \\ Th. de GRAAUW \\ Laboratory for Space Research Groningen \\ University of Groningen \\ PO Box 800 \\ NL-9700 AV Groningen \\ The Netherlands
}

\section{Introduction}

In this short review we describe recent new observations of millimetre transitions of molecules in selected regions of the Magellanic Clouds. The observations were made using the Swedish-ESO Submillimetre Telescope, SEST, (Booth et al. 1989), the relatively high resolution of which facilitates, for the first time, observations of individual giant molecular clouds in the Magellanic Clouds. We have mapped the distribution of the emission from the two lowest rotational transitions of ${ }^{12} \mathrm{CO}$ and ${ }^{13} \mathrm{CO}$ and hence have derived excitation conditions for the molecule. In addition, we have observed several well-known interstellar molecules in the same regions, thus doubling the number of known molecules in the Large Magellanic Cloud (LMC). The fact that all the observations have been made under controlled conditions with the same telescope enables a reasonable intercomparison of the molecular column densities. In particular, we are able to observe the relative abundances among the different isotopically substituted species of $\mathrm{CO}$.

\section{Observations of Carbon monoxide}

\subsection{MULTI-LINE STUDIES AND OPTICAL DEPTH}

Booth and Johansson (1990) and Israel and de Graauw (1990) have described new observations of the CO $(\mathrm{J}=1-0)$ rotational transition in the Magellanic Clouds using the Swedish-ESO Submillimetre Telescope, SEST. Johansson et al. (in prep.) have made further SEST observations of the two lowest rotational transitions of ${ }^{12} \mathrm{CO}$ and ${ }^{13} \mathrm{CO}$. The regions observed are the four clouds in the vicinity of 30 Dor mapped by Johansson et al. (1989) and identified in Fig. 1 in the paper by Booth and Johansson. The CO cloud contour plots are reproduced here in Fig. 1 together with the spectra centred on each of four regions, three associated with N159 and a fourth with 30 Dor. We have made 5-point maps in the (2-1) transitions and so have been able to estimate the $\mathrm{CO}(2-1)$ emission expected in the (1-0) beam. The observed antenna temperatures have been converted to main beam brightness temperatures. 
An analysis of these data shows that the ${ }^{12} \mathrm{CO} /{ }^{13} \mathrm{CO}$ line ratios are of order 1.15 compared with values of 1.5-2 which are typical for Galactic giant molecular clouds. This implies a lack of saturation in the ${ }^{12} \mathrm{CO}(1-0)$ line in these LMC molecular clouds.

Both a simple analysis of the line ratios assuming LTE, and a standard large velocity gradient (LVG) solution give values of the ${ }^{12} \mathrm{CO}(1-0)$ optical depth close to unity, kinetic temperatures in the range $10-40 \mathrm{~K}$ and isotopic ratios, ${ }^{12} \mathrm{CO} /{ }^{13} \mathrm{CO}$ in the range $10-30$. Column densities of ${ }^{12} \mathrm{CO}$ are in the range $10^{16.5}$ to $10^{17.4}$, significantly lower than for Galactic molecular clouds of similar sizes. This fact is also reflected in the low optical depths. (For a fuller description of this analysis see Johansson 1990).

Because the ${ }^{12} \mathrm{CO}(1-0)$ lines in the LMC indicate much lower optical depths than in the Galaxy, Booth and Johansson (1990) suggest that the conversion factor between CO luminosity and molecular hydrogen mass may be greater in the LMC than in the Galaxy.
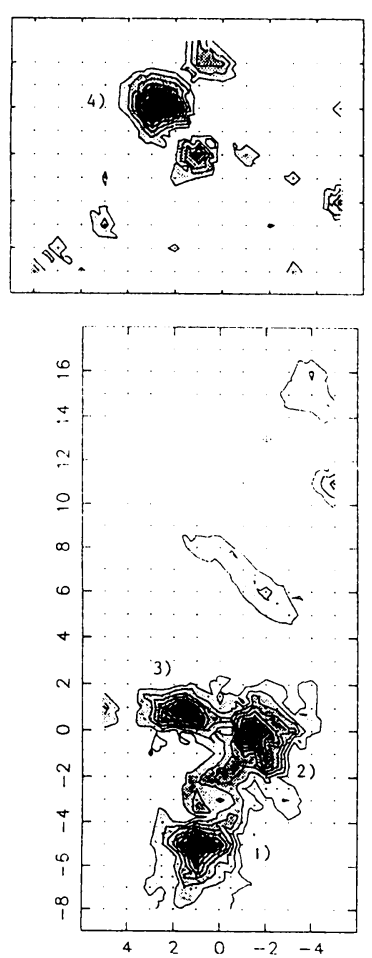

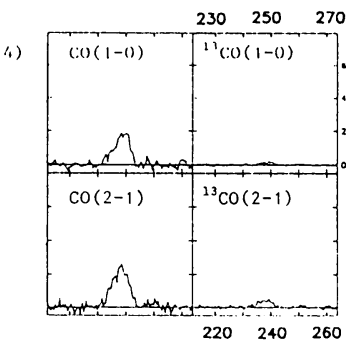

3)

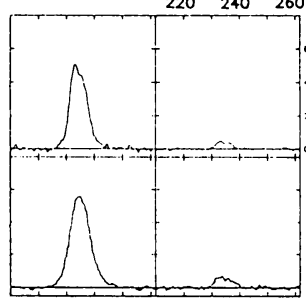

$220 \quad 240 \quad 260$

1)

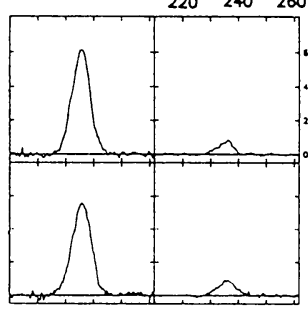

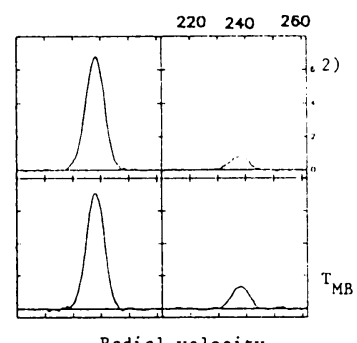

Radial velocity

Figure 1. Spectra of ${ }^{12} \mathrm{CO}$ and ${ }^{13} \mathrm{CO}$ in the two lowest rotational transitions in the three regions of N159 $(1,2,3)$ and near 30 Dor $(4)$.

\subsection{CO ISOTOPE RATIOS}

The ${ }^{12} \mathrm{CO} /{ }^{13} \mathrm{CO}$ isotope abundance ratios, determined for the four clouds observed, are given in Table 1.

In $\mathrm{N} 159$ position 2 we have also detected $\mathrm{C}^{18} \mathrm{O}$ in its two lowest rotational transitions. The $\mathrm{C}^{18} \mathrm{O}$ isotopic ratios are also tabulated in Table 1 . The ratio $\mathrm{T}_{\mathrm{MB}}\left({ }^{13} \mathrm{CO}\right) / \mathrm{T}_{\mathrm{MB}}\left(\mathrm{C}^{18} \mathrm{O}\right)=20 \pm 5$, 
averaged over the two transitions. This should be close to the true isotopic ratio, $\left[{ }^{13} \mathrm{CO}\right] /\left[\mathrm{C}^{18} \mathrm{O}\right]$, since the transitions are optically thin, (but see below).

Table 1. CO isotope ratios.

\begin{tabular}{llccccc}
\hline & & pos 1 & $\begin{array}{c}\text { N159 } \\
\text { pos 2 }\end{array}$ & pos 3 & 30 DOR & $\begin{array}{c}\text { "LMC" } \\
\text { (mean) }\end{array}$ \\
${ }^{12} \mathrm{CO} /{ }^{13} \mathrm{CO}$ & & $12-50$ & $11-19$ & $12-25$ & $14-22$ & $17 \pm 3$ \\
& & & 25 & & & \\
${ }^{13} \mathrm{CO} / \mathrm{C}^{18} \mathrm{O}$ & $\mathrm{J}=1-0$ & & 14 & & & $20 \pm 5$ \\
${ }^{12} \mathrm{CO} / \mathrm{C}^{18} \mathrm{O}$ & $\mathrm{J}=2-1$ & & 350 & & & $350 \pm 150$ \\
\hline
\end{tabular}

The CO isotope ratios given in Table 1 may be compared with the Galactic isotopic ratios ${ }^{12} \mathrm{C} /{ }^{13} \mathrm{C}$ and ${ }^{16} \mathrm{O} /{ }^{18} \mathrm{O}$ ratios derived by Langer et al. (1984) for the Galaxy by modelling, to account for chemical fractionation:

Galactic Centre

Galactic ring clouds

$$
\begin{gathered}
{\left[{ }^{12} \mathrm{C}\right] /\left[{ }^{13} \mathrm{C}\right]} \\
\sim 25 \\
\sim 100
\end{gathered}
$$

$$
\begin{gathered}
{\left[{ }^{16} \mathrm{O}\right] /[18 \mathrm{O}]} \\
\sim 350 \\
\sim 700
\end{gathered}
$$

The Galactic gradient in ${ }^{12} \mathrm{C} /{ }^{13} \mathrm{C}$ has also been addressed in a recent paper by Langer and Penzias (1990) with similar results. Langer et al. (1984) how that ${ }^{13} \mathrm{CO}$ can be significantly enhanced by fractionation through the reaction:

$$
{ }^{13} \mathrm{C}+{ }^{12} \mathrm{CO}={ }^{13} \mathrm{CO}+{ }^{12} \mathrm{C}^{+}+\Delta \mathrm{E} .
$$

The ratio $\left[{ }^{12} \mathrm{CO}\right] /\left[{ }^{13} \mathrm{CO}\right]$ is dependent on temperature, density and metallicity, in the sense that as temperature and density increase and metal abundance decreases the ${ }^{12} \mathrm{CO} /{ }^{13} \mathrm{CO}$ ratio approaches the ${ }^{12} \mathrm{C} /{ }^{13} \mathrm{C}$ ratio. The metal abundance in the $\mathrm{LMC}$ is significantly lower than in the Galaxy, so if total densities and temperatures are similar to those of Galactic clouds, fractionation may be less significant in the LMC. The model by Langer et al. does not take radiation fields into account, however. The effect of the lower metal abundances in the LMC and SMC is such that shielding by dust, and even self-shielding by the $\mathrm{CO}$ molecules is reduced relative to the Galaxy. Thus, photodissociation becomes important and isotope-selective photodissociation competes with the ${ }^{13} \mathrm{CO}$ fractionation reactions in establishing the relative abundance of $\left[{ }^{12} \mathrm{CO}\right] /\left[{ }^{13} \mathrm{CO}\right]$, particularly near the LMC HII regions studied in this work. (See van Dishoeck and Black (1988) and Maloney and Black (1988) for a discussion of the effects of photodissociation in the Magellanic Clouds.)

In contrast to ${ }^{13} \mathrm{CO}$, the oxygen isotopic fractionation is small in $\mathrm{C}^{18} \mathrm{O}$, so we might expect that the $\left[{ }^{12} \mathrm{C}^{16} \mathrm{O}\right] /\left[{ }^{12} \mathrm{C}^{18} \mathrm{O}\right]$ ratio may be a good estimate of the ratio $\left[{ }^{16} \mathrm{O}\right] /\left[{ }^{18} \mathrm{O}\right]$. However, we note that a recent re-axamination of the 'double isotope ratio', $\left[{ }^{13} \mathrm{CO}\right] /\left[\mathrm{C}^{18} \mathrm{O}\right]$ for Galactic molecular clouds by Taylor and Dickman (1989) reveals large increases in the ratio of the column densities of the two species at low extinctions which these authors attribute to isotopic fractionation and isotope-selective photodissociation. Thus, even the oxygen isotope abundance ratio may be difficult to determine directly from the observational data. 


\section{Other molecules in the LMC}

The number of molecules detected in the Magellanic Clouds has been doubled through recent observations with SEST, (Johansson et al. in prep., de Graauw et al. in prep.). In Table 2 we list the molecules detected before 1988. These data have been obtained with a variety of telescopes with different beam sizes and in many cases the emission involves anomalous excitation, which complicates comparison of molecular abundances.

Table 2. Molecules detected in the Magellanic Clouds (before 1988).

\begin{tabular}{|c|c|c|c|c|}
\hline Molecule & $\begin{array}{l}\text { Frequency } \\
(\mathrm{GHz})\end{array}$ & $\begin{array}{c}\text { Beamsize } \\
\text { (arcmin) }\end{array}$ & Region & Reference \\
\hline $\mathrm{H} 2$ & IR & & LMC,SMC & $\begin{array}{l}\text { Koornneef \& Israel } 1985 \text {, } \\
\text { Israel \& Koornneef } 1988\end{array}$ \\
\hline $\mathrm{OH}$ & 1.7 & 12.4 & LMC & Gardner 1984 (rev.) \\
\hline $\mathrm{CH}$ & 3.3 & 6.4 & LMC & Whiteoak et al. 1980 \\
\hline $\mathrm{CO}$ & 115.0 & $\sim 4.0$ & LMC,SMC & Israel 1984 (rev.) \\
\hline (single pos) & 230.0 & $\sim 2.0$ & LMC,SMC & Israel et al. 1986 \\
\hline CO & 115.0 & 8.6 & LMC & Cohen et al. 1988 \\
\hline (mapping) & & & SMC & Rubio et al. 1990 \\
\hline $\mathrm{HCO}+$ & 89.0 & 3.3 & $\mathrm{LMC}$ & Batchelor et al. 1981 \\
\hline $\mathrm{H}_{2} \mathrm{CO}$ & 4.8 & 4.3 & LMC & Whiteoak \& Gardner 1976 \\
\hline $\begin{array}{l}\text { OH } \\
\text { (MASER) }\end{array}$ & 1.7 & 1.7 & LMC & $\begin{array}{l}\text { Caswell \& Haynes } 1981 \\
\text { Haynes \& Caswell } 1981\end{array}$ \\
\hline $\begin{array}{l}\mathrm{H}_{2} \mathrm{O} \\
\text { (MASER) }\end{array}$ & 22.0 & & LMC,SMC & $\begin{array}{l}\text { Scalise \& Braz } 1982 \\
\text { Whiteoak et al. } 1983 \\
\text { Whiteoak \& Gardner } 1986\end{array}$ \\
\hline
\end{tabular}

The molecules detected in the new surveys using SEST are listed in Table 3. The searches have, so far, concentrated on region 2 (Fig. 1) in the LMC and the HII region, LIRS 49 (position from Schwering 1988) in the SMC. As shown in Table 3, relatively few molecules are seen in the SMC, and those lines which are detected are a factor of $\sim 6$ weaker than the equivalent lines in the LMC.

Molecules like CS and HCO+ usually reflect high densities $\left(>10^{4}\right)$, so, even though CO has a low optical depth, high density clumps must be present in the molecular clouds in the Magellanic Clouds. Note, however, that molecules like CS and $\mathrm{HCO}+$, because of their large dipole moments, may suffer excitation by electrons at lower neutral densities when the ionization fraction is high (J. Black, private communication).

Using the SEST data, we have attempted to calculate molecular abundances. In doing so, we have made the following assumptions: i) the detected molecular lines have low optical depths; ii) a Boltzmann distribution of energy states applies, defined by a temperature in the range $10-50 \mathrm{~K}$ (it is difficult to be more precise); and iii) the spatial extent of the molecules is similar, relative to $\mathrm{CO}$, to that observed in the Galaxy. Here we have adopted radial distributions varying from one tenth of the CO size up to the same extent. In the case of SO there is compelling evidence that in Galactic clouds it is much more compact than $\mathrm{CO}$, hence we have adopted a range of sizes varying from 0.01 to 0.1 that of the $\mathrm{CO}$ cloud. 
Table 3. Molecular lines detected with SEST

\begin{tabular}{lrll}
\hline MOLECULE & $\begin{array}{c}\text { FREQUENCY } \\
\text { GHz }\end{array}$ & $\begin{array}{l}\text { LMC } \\
\mathrm{T}_{\mathrm{MB}}(\mathrm{K})\end{array}$ & $\begin{array}{l}\text { SMC } \\
\mathrm{T}_{\mathrm{MB}}(\mathrm{K})\end{array}$ \\
\hline $\mathrm{CS}(2-1)$ & 98 & 0.30 & 0.05 \\
$\quad(5-4)$ & 245 & 0.14 & - \\
$\mathrm{CN}(1-0)$ & 113 & 0.11 & $<0.03$ \\
$\quad(2-1)$ & 227 & 0.11 & - \\
$\mathrm{SO}\left(3_{2}-2_{1}\right)$ & 99 & 0.08 & - \\
$\mathrm{HCO}+(1-0)$ & 89 & 0.48 & 0.07 \\
$\mathrm{HCN}(1-0)$ & 89 & 0.16 & $<0.02$ \\
$\mathrm{HNC}(1-0)$ & 91 & 0.12 & - \\
$\mathrm{H}_{2} \mathrm{CO}\left(3_{1,2}-2_{1,1}\right)$ & 226 & 0.12 & - \\
$\mathrm{C}_{3} \mathrm{H}_{2}\left(2_{1,2}-1_{0,1}\right)$ & 85 & 0.09 & $<0.08$ \\
$\mathrm{C}_{2} \mathrm{H}(1-0)$ & 87 & 0.10 & - \\
\hline
\end{tabular}

The molecular abundances relative to $\mathrm{CO}$ are shown in Fig. 2, in which a comparison is also made with two Galactic clouds, Orion and TMC-1 (Johansson 1990). It is difficult, because of the assumptions, to derive accurate abundances from the new data but one can note the trends, i.e. that abundances relative to CO scale in the same way as in the Galaxy. Note that, with the exception of SO, all the molecules observed are carbon bearing.

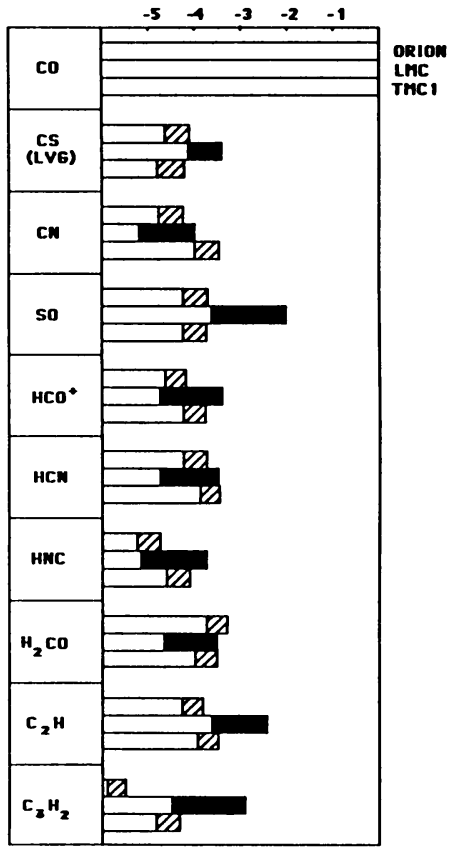

Figure 2. Log of abundance relative to $\mathrm{CO}$ in the LMC (position 2) compared with molecular abundances in the Galactic Clouds OMC-1 and TMC-1. 


\section{Acknowledgments}

The authors are grateful to Lars E.B. Johansson for providing material before publication and for help in the preparation of this manuscript, and to John Black for critical comments.

\section{References}

Batchelor, R.A., McCulloch, M.G., Whiteoak, J.B. (1981), M.N.R.A.S. 194, 911.

Booth, R.S., Delgado, G., Hagström, M., Johansson, L.E.B., Murphy, D.C., Olberg, M., Whyborn, N.D., Greve, A., Hansson, B., Lindström, C.O., Rydberg, A. (1989), Astron. Astrophys. 216, 315.

Booth, R.S., Johansson, L.E.B. (1990), these proceedings.

Caswell, J.L., Haynes, R.F. (1981), M.N.R.A.S. 194, 33P.

Cohen, R.S., Dame, T.M., Garay, G., Montani, J., Rubio, M., Thaddeus, P. (1988), Astrophys. J. 331, L95.

Gardner, F.F. (1984), IAU Symp. 108 Structure and Evolution of the Magellanic Clouds, S. van den Bergh, K.S. de Boer (eds.) (Reidel: Dordrecht) p.397.

Haynes, R.F., Caswell, J.L. (1981), M.N.R.A.S. 197, 23P.

Israel, F.P. (1984), IAU Symp. 108 Structure and Evolution of the Magellanic Clouds, S. van den Bergh, K.S. de Boer (eds.) (Reidel: Dordrecht) p.319.

Israel, F.P., de Graauw, Th., (1990), these proceedings.

Israel, F.P., de Graauw, Th., van de Stadt, H., de Vries, C.P. (1986), Astrophys. J. 303, 186.

Israel, F.P., Koornneef, J. (1988), Astron. Astrophys. 190, 21.

Johansson, L.E.B. (1990), IAU Symp. 146 Dynamics of Galaxies, F. Combes (ed.) in press.

Johansson, L.E.B., Booth, R.S., Murphy, D.C., Olberg, M. (1989), Submillimetre Astronomy, G.D. Watt, A.S.Webster (eds.) (Kluwer Academic Publishers).

Koornneef, J., Israel, F.P. (1985), Astrophys. J. 291, 156.

Langer, W.D., Graedel, T.E., Frerking, M.A., Armentrout, P.B. (1984), Astrophys. J. 277, 581.

Langer, W.D., Penzias, A. (1990), Astrophys. J., 357, 477.

Maloney, P., Black., J.H. (1988), Astrophys. J. 325, 389.

Rubio, M., Garay, G., Montani, J., Thaddeus, P. (1990), Astrophys. J. (submitted).

Scalise, E., Braz, M.A. (1982), Astron. J. 87, 528.

Schwering, P.B.W. (1988), PhD thesis, Univ. Leiden.

Taylor, D.K., Dickman, R.L. (1989), Astrophys. J. 341, 293.

van Dishoeck, E.F., Black, J.H. (1988), Molecular Clouds in the Milky Way and External Galaxies, R.L. Dickman, R.L. Snell, J.S. Young (eds.) (Springer-Verlag).

Whiteoak, J.B., Gardner, F.F. (1976), M.N.R.A.S. 174, 51P.

Whiteoak, J.B., Gardner, F.F. (1986) M.N.R.A.S. 222, 513.

Whiteoak, J.B., Gardner, F.F., Höglund, B. (1980), M.N.R.A.S. 190, 17P.

Whiteoak, J.B., Wellington, K.J., Jauncey, D.L., Gardner, F.F., Forster, J.R., Caswell, J.L., Batchelor, R.A. (1983), M.N.R.A.S. 205, 275. 